\title{
DU MARTIN-PECHEUR A LA MANTE RELIGIEUSE
}

\author{
DO MARTIM-PESCADOR AO LOUVA-A-DEUS
}

Jean Libis ${ }^{1}$

\section{RÉSUMÉ}

Force est de constater que Gaston Bachelard et Roger Caillois ne sont guère convoqués par ce que l'on a appelé parfois la French Philosophy. Ce sont pourtant deux penseurs profondément originaux et qui se tiennent à la lisière de la cartographie philosophique. Curieusement, ils sont d'abord présents l'un et l'autre dans la création de la revue Inquisitions qui paraît en 1936 et dont un seul numéro connaîtra le chemin de la publication. Plus tard leurs chemins vont nettement différer et les références de Bachelard à Caillois se limiteront surtout aux traités sur le mythe et sur le sacré. L'un et l'autre vont cependant côtoyer des préoccupations souvent communes: le destin de la poésie, les ambitions de la science, la splendeur des règnes minéraux et végétaux. Il faut $\mathrm{y}$ ajouter une relation subtile à la question métaphysique du sens et à sa réduction éventuelle. En tout cela d'ailleurs, les différences d'approches l'emportent sans doute sur les analogies. Peu nous importe: nous avons là affaire à deux cheminements solitaires, d'une puissance hors du commun.

Mots-clés: Inquisitions. Mélancolie. Dualisme.

\footnotetext{
${ }^{1}$ Professeur de Philosophie à Dijon (France). Ancien président de l'Association des amis de Gaston Bachelard.
} 


\section{DU MARTIN-PECHEUR A LA MANTE RELIGIEUSE \\ Jean Libis}

\section{RESUMO}

É claro que Gaston Bachelard e Roger Caillois quase não são convocados pelo que às vezes se chamou de French Philosophy. Entretanto, são dois pensadores certamente originais e que ficam à beira da cartografia filosófica. Curiosamente, eles estão inicialmente presentes na criação da revista Inquisitions que surge em 1936 e da qual apenas um número da revista é publicado. Mais tarde seus caminhos se distanciam claramente, e as referências de Bachelard para Caillois se limitarão sobretudo aos tratados sobre mitos e sobre o sagrado. Os dois irão, contudo, cotejar preocupações frequentemente comuns: o destino da poesia, as ambições da ciência, o esplendor dos reinos minerais e vegetais. É necessário adicionar a essa lista uma relação sutil sobre a questão metafísica do sentido e sua eventual redução. Além disso, as diferenças de abordagens talvez superem as analogias. Pouco nos importa: lidamos aqui com dois caminhos solitários, de uma força fora do comum.

Palavras-chave: Inquisitions. Melancolia. Dualismo.

$$
* * *
$$

Toute étude comparative entre deux auteurs comporte une part d'artifice, qu'il faut s'efforcer de réduire. Gaston Bachelard (1884-1962) et Roger Caillois (1913-1978) sont d'abord deux penseurs d'une puissante originalité, tant dans leurs aperçus philosophiques que dans la constitution de leurs œuvres. On remarquera d'abord que le panthéon philosophique les tient à distance, non sans quelque raison peut-être. Ont-ils d'ailleurs élaboré ce qu'il convient d'appeler une philosophie à part entière? Car ils ont exploré, surtout le second, des champs d'investigation ouverts et déroutants. Dans un cas comme dans l'autre, le végétal, l'animal, le minéral constituent des objets de fascination à part entière, diversement conjugués de l'un à l'autre. Dans leurs ouvrages la poésie et la science forment un contrepoint parfois complexe, qu'il s'agira de démêler: tâches qui s'avéreront d'un auteur à l'autre assez différentes. Si Bachelard a 


\section{DU MARTIN-PECHEUR A LA MANTE RELIGIEUSE \\ Jean Libis}

relativement peu cité Caillois dans ses livres ${ }^{2}$, en revanche les auteurs se sont d'abord rencontrés dan sun lieu précis, moins géographique que culturel et éditorial: à savoir l'élaboration d'une revue dont l'histoire à elle seule mérite un temps d'arrêt.

\section{INQUISITIONS}

En 1936 paraît une revue dont le titre est provocateur: Inquisitions ${ }^{3}$. Sa destinée sera brève puisque cette revue paraîtra dan sun numéro unique, qui n'aura pas de suite. L'équipe de direction se compose de Louis Aragon, Roger Caillois, Jules Monnerot et Tristan Tzara. Singulière équipe à dire vrai, dont l'éclatement peut sembler a priori inévitable tant elle s'avère résolument éclectique. Les deux premiers articles sont signés respectivement par Gaston Bachelard et Roger Caillois lui-même, ce qui nous place au cœur même de notre sujet. D'après Henri Béhar, Caillois s'est flatté d'avoir obtenu la collaboration du philosophe après une rencontre dans les Vinarny de Prague!

L'article de Bachelard, intitulé significativement Le surrationalisme, constitue un véritable manifeste de l'épistémologie bachelardienne dans son ensemble: passée, présente et à venir. Contre un rationalisme classique et somme toute paresseux, Bachelard prône une rationalité ouverte, turbulente, allant même jusqu'à déclarer que «dans le règne de la pensée, l'imprudence est une méthode ». Plus surprenant, il accompagne cette apologie d'une citation de Nietzsche, assez intempestive dans ce lieu, il faut bien l'avouer. En fin de parcours, Bachelard prône la nécessité d'un rationalisme ouvert, au sein duquel la raison lutte avec elle-même et nourrit sa propre dialectique interne. On peut lire les travaux épistémologiques ultérieurs du philosophe à la lumière de cette programmation fondamentale qui était déjà clairement en jeu dans un ouvrage comme Le nouvel esprit scientifique (1934).

Le texte de Caillois qui vient juste ensuite s'intitule Pour une orthodoxie militante: les tâches immédiates de la pensée moderne. Ce texte est très curieux à la fois par la révolte qu'il exprime contre un monde moderne avachi, par l'idée d'un

\footnotetext{
${ }^{2}$ Les trois références à Caillois sont les suivantes: Lautréamont (BACHELARD, 1939, p. 144); La philosophie du non (BACHELARD, 1940, p. 59); et L'eau et les rêves (BACHELARD, 1942, p. 181).

${ }^{3}$ La revue Inquisitions a été rééditée en fac-similé en 1990 aux Editions du CNRS, avec une riche présentation de Henri Béhar à laquelle je suis ici très redevable. Je souhaite aussi remercier Jacques Poirier qui m'a permis de prendre connaissance de cette revue unique en son genre.
} 


\section{DU MARTIN-PECHEUR A LA MANTE RELIGIEUSE \\ Jean Libis}

redressement possible et salutaire, enfin par son ambition surprenante. Il se propose « de mettre en œuvre la totalité de l'être ... ». De façon ici inattendue, il cite également un mot de Nietzsche, «Devenez âpres » et se réfère au Nouvel esprit scientifique de Bachelard. Le ton est très polémique et il faut prendre le mot «orthodoxie » dans son sens durement étymologique. Le surréalisme lui-même, mouvement de révolte s'il en est, est traité avec sévérité et pour finir est répudié pour son caractère devenu «maniaque et purement rituel».

La juxtaposition de ces deux articles dans une revue avant-gardiste qui n'aura eu qu'une existence brève constitue un phénomène des plus singuliers. Si l'idée d'une refonte intellectuelle, voire d'une révolution culturelle, est commune aux deux auteurs, Bachelard situe son propos dans le champ de la seule activité scientifique, alors que Caillois opère une généralisation à la fois ambitieuse et quelque peu abstraite. De fait il y a là en ce point de départ un élément symptomatique de ce que seront les évolutions ultérieures de nos deux complices d'apparence. Bachelard va pratiquement circonscrire sa réflexion, d'abord à l'activité de la raison scientifique, ensuite à une exploration, psychanalytique, puis phénoménologique, de l'imagination poétique. Caillois sera tenté par un champ beaucoup plus large et presque indéfini: de sorte que la politique, l'histoire et la minéralogie feront aussi partie de ses investigations qui nous vaudront des pages fascinantes et aventureuses.

\section{DES PANORAMAS HETEROGENES}

Les œuvres de Roger Caillois et de Gaston Bachelard occupent l'une comme l'autre un espace éditorial important et couvrent de vastes panoramas. La première compte environ 30 volumes publiés et la seconde en comporte exactement 25 si l'on y intègre les Fragments d'une poétique du feu,inachevés du vivant de l'auteur et publiés à titre posthume avec l'accord de Suzanne Bachelard.

Toutefois les champs d'investigation de ces auteurs sont assez différents dans leurs structures, leurs contenus et leurs ambitions. C'est surtout l'œuvre de Caillois qui semble hantée par un désir illimité de prospection, de compréhension et d'induction généralisée. Deux livres essentiels dessinent les fondements d'une anthropologie 


\section{DU MARTIN-PECHEUR A LA MANTE RELIGIEUSE \\ Jean Libis}

constitutive d'un projet à long terme: Le mythe et l'homme (1938) bientôt suivi de L'homme et le sacré (1939). Ce sont d'ailleurs ces deux ouvrages qui attirent l'intérêt de Gaston Bachelard, notamment dans un passage de Lautréamont et dans un autre de L'eau et les rêves. Ils constituent une sorte de diptyque homogène qui vise à saisir la nature fondamentale des sociétés sans écriture: celle qui s'articule sur le mythe, le rite, la fête, le sacrifice, et surtout lie indissolublement les conduites sexuelles avec la sacralité, ainsi que les instincts de mort avec les instincts de vie. De telle sorte qu'ils brossent l'édifice d'une véritable anthropologie qui pourrait se résumer ainsi: l'homme est un animal mythogène dont les pulsions sexuelles sont réglées par une religiosité, certes variable culturellement, mais transcendantale dans son essence profonde.

À partir de là, l'œuvre de Caillois va s'élargir avec une liberté parfois déconcertante. Génial touche-à-tout, comme l'écrira Michel Panoff, Caillois va explorer aussi bien l'entomologie que l'histoire (Le rocher de Sisyphe), la politique (Description du marxisme), la littérature dans tous ses états, et la minéralogie, notamment dans L'écriture des pierres. L'œuvre est foisonnante, multiple, souvent très inventive, parfois un peu débridée et épistémologiquement incertaine. Elle bourgeonne aussi vers le récit de voyage - l'évocation de la Patagonie est un grand moment d'anthologie - et surtout vers deux ouvrages tardifs qui constituent un bilan philosophique et autocritique sans concession, nous entraînant vers un scepticisme croissant et iconoclaste: Cases d'un échiquier et Le fleuve Alphée.

En regard, le parcours de Bachelard apparaît comme paradoxalement plus resserré et, avouons-le, plus homogène malgré une diversité apparente qui souvent déconcerte le lecteur néophyte. Il s'enracine dans une culture scientifique où la physique quantique et la chimie des particules constituent les ingrédients fondamentaux qui vont pousser le philosophe à élaborer une épistémologie originale et dynamique. C'est seulement à partir de La psychanalyse du feu (1938) que Bachelard va explorer sciemment une deuxième voie qui sera celle de l'imagination littéraire, ou, plus précisément, celle de l'image poétique. Ces deux voies seront désormais frayées en parallèle et s'écarteront toujours davantage l'une de l'autre. Il importe cependant de noter que la trajectoire bachelardienne comporte deux parenthèses: celle d'une métaphysique non-bergsonienne du temps, illustrée par L'intuition de l'instant (1932) et 


\section{DU MARTIN-PECHEUR A LA MANTE RELIGIEUSE \\ Jean Libis}

La dialectique de la durée (1936); et celle, énigmatique, inclassable, d'une étude consacrée à Lautréamont (1939). Pour être complet, il convient aussi de mentionner les divers articles, dispersés, dans lesquels Bachelard présente les travaux de peintres ou de sculpteurs qu'il relie à sa propre philosophie de l'imaginaire.

Disons, d'une façon synthétique, que Bachelard n'a pas la même ambition anthropologique que Caillois. Il n'y a pas chez lui une théorie achevée du mythe, ni une approche systématique des sociétés archaïques. D’une façon plus générale, la religion, la politique ${ }^{4}$, l'histoire sont pratiquement absentes de son travail philosophique. Bien que certains commentateurs aient parlé de son anthropologie, cette dernière me paraît délibérément inachevée et prudemment retenue par un dialogue avec les images poétiques. En dehors de sa métaphysique du temps, qui peut être considérée comme de nature spécifiquement philosophique, il étudie essentiellement la raison scientifique en devenir et l'imagination poétique dans son foisonnement intemporel. Et certes les œuvres de Bachelard et de Caillois peuvent se rencontrer sur un certain nombre de points: précisément sur celui de l'image poétique et de la référence à l'imaginaire; et sur celui d'une méfiance à l'égard de la philosophie institutionnelle. Mais le cheminement du second comporte une sorte de boursouflure baroque et fascinante, qui le conduit à un travail d'explorateur audacieux et parfois risqué. Bachelard, lui, n'envisage pas de penser l'univers.

\section{LA FASCINATION DE L'UNITE ET L'EXPLORATION DU DUALISME}

Sur l'antique question métaphysique de l'un et du multiple, ou plus précisément ici de l'unité et de la dualité, la comparaison entre les œuvres de Roger Caillois et Gaston Bachelard prend davantage de signification. Elle fait apparaitre deux démarches en quelque sorte inverses l'une de l'autre, sans toutefois que l'une ne s'oppose frontalement à l'autre. En d'autres termes: elles se déploient à peu de choses près indépendamment l'une de l'autre.

\footnotetext{
${ }^{4}$ Robert Damien a cependant tenté de montrer qu'on peut diagnostiquer en filigrane une présence originale du facteur politique dans l'œuvre bachelardienne.
} 


\section{DU MARTIN-PECHEUR A LA MANTE RELIGIEUSE \\ Jean Libis}

Roger Caillois semble taraudé par une curiosité insatiable et voyageuse qui l'amène constamment à franchir des frontières, à tisser des passerelles, à risquer des métaphores. Il est, comme le notent André-Alain Morello et d'autres commentateurs, fasciné par les analogies (MORELLO, 2013, p. 9). N'affirme-t-il pas qu'il y a continuité entre la matière et l'imagination? Les pages extraordinaires qu'il consacre au mimétisme des insectes, et notamment l'analogie qu'il tisse entre l'attitude figée de la mante religieuse et le masque utilisé dans les rites de nombreuses sociétés archaïques, sontici particulièrement révélatrices. Tout se passe comme si une conduite humaine complètement ritualisée était la transposition directed'une attitude empruntée au règne animal et qui plus est au monde élémentaire des insectes. D’ailleurs ne soutient-il pas, dans Le mythe et l'homme, que le mythe et l'instinct des insectes s'apparenteraient à un processus identique, qui les enracinerait somme toute dans une même nature, une même instance pulsionnelle? Idée fascinante s'il en est, qui toutefois semble procéder davantage d'une induction inspirée que d'une démarche proprement scientifique. On la trouve cependant réitérée dans plusieurs cas et toujours mue par une sorte d'inspiration heuristique. Dans un autre ouvrage, c'est le monde végétal qui est requis pour une série de comparaisons envoûtantes. «Je découvris au Brésil, écrit l'auteur, la végétation par excellence, dont la puissance redoutable balance celle de l'homme... ». Et un peu plus loin: «La végétation semble redoutable par d'inépuisables pouvoirs occultes, dont l'étendue et la variété ne cessent de s'accroître $\nu^{5}$. Enfin l'incursion opérée par Caillois dans l'univers de la minéralogie et de ses graphismes fantastiques constitue une autre démarche audacieuse qui invoque «l'écriture » des pierres dans un livre à tous les égards original.

Tout se passe comme si Caillois était fasciné par l'idée générale d'une unité de la nature qui demeure cependant pour lui davantage un schème transcendantal que l'objet d'une démonstration impossible à établir rigoureusement. C'est pourquoi l'auteur est souvent en proie à des hésitations, pour ne pas dire à des contradictions. S'il affirme à plusieurs reprises l'unité du monde et de la nature, il reconnait dans la préface de Cases d'un échiquier qu'il échoue somme toute à en dresser véritablement

\footnotetext{
${ }^{5}$ Ces deux citations sont extraites du chapitre 6 dans Le fleuve Alphée (CAILLOIS, 1978, p. 126 et 139). Le chapitre lui-même s'intitule de façon significative « La condition végétale ».
} 


\section{DU MARTIN-PECHEUR A LA MANTE RELIGIEUSE \\ Jean Libis}

l'ossature: «Je rêve que ces pages procurent l'image lacunaire et briséed'une représentation du monde qui n'a pas trouvé son harmonie et que je ne suis pas parvenu à fixer » (CAILLOIS, 1970, p. 10). Si le tableau des 92 éléments de Mendéléieff semble attester d'un ordre immanent à la matière, il déclare néanmoins dans la même Préfaceque l'harmonie du monde n'a pas été trouvée. L'œuvre de Caillois présente ainsi comme une hésitation théorique fondamentale. Son indiscutable désir d'unité se nourrit de signes (la rose des sables, le goliath, la mante religieuse), mais tout autant qu'elle les suscite. Ce désir n'est d'ailleurs pas de nature mystique mais bien plutôt de nature cosmologique ${ }^{6}$. Caillois rêve d'un cosmos dans lequel régneraient d'innombrables correspondances porteuses d'harmonies. L'observation peut servir cette vision des choses tout autant qu'elle peut la desservir. De ce point de vue, ses inductions audacieuses sont souvent tempérées par une prudence épistémologique de principe (ce qui indirectement nous conduit vers Bachelard). Guillaume Bridet a pu écrire qu'il y a chez Caillois une sorte de dialectique entre un dualisme combattif et un monisme pacificateur. Ainsi le jeu d'échecs lui permet de « penser l'unité sans sacrifier la dualité »(BRIDET, 2013, p. 91): l'unité des règles du jeu et la dualité des blancs et des noirs.

Très clairement Gaston Bachelard se situe dans une perspective qui, à l'inverse de celle de Caillois, éloigne le rêve d'unité.Dans ce livre central et paradigmatique qu'est La formation de l'esprit scientifique,le philosophe répète que l'idée d'unité est captieuse, qu'elle engendre des inductions illégitimes. On peut ici aisément multiplier les références. Ainsi: «... Le progrès scientifique marque ses plus nettes étapes en abandonnant les facteurs philosophiques d'unification facile tels que l'unité d'action du Créateur, l'unité de plan de la nature, l'unité logique »(BACHELARD, 1938, p. 16). Plus nette encore, plus vigoureuse, plus polémique est cette formulation: «Il n'y a plus guère que des philosophes pour chercher, sinon la pierre philosophale, du moins l'idée philosophale qui expliquerait le monde »(BRIDET, 2013, p. 94). En définitive l'idée d'unité est assimilée à un obstacle épistémologique puissant qui fait l'objet d'une critique vigoureuse tout au long du cinquième chapitre. Elle relève de la logique du désir et non d'un constructivisme objectif. Caillois a bien subodoré cela, mais il

\footnotetext{
${ }^{6}$ Cependant, tout à la fin du Fleuve Alphée, il se risque à l'expression de «Mystique matérialiste » (CAILLOIS, 1978, p. 214).
} 


\section{DU MARTIN-PECHEUR A LA MANTE RELIGIEUSE \\ Jean Libis}

demeure, nous l'avons vu, dans une certaine hésitation. Pour Bachelard ni le monde ni la nature n'existent comme tels en soi. Et cette vision des choses apparaît particulièrement dans un article intitulé: Univers et réalité (BACHELARD, 1972, p. 104). Avec son humour habituel, Bachelard se présente comme un philosophe, malheureux, qui a perdu son monde. De fait: qui a perdu le monde. Car les physiciens et les chimistes contemporains ne lui proposent pas un univers, mais plus modestement un pluralisme cohérent. La connaissance scientifique procède par zones, par régions, par départements; alors que l'idée d'univers draine constamment avec elle une sorte de transcendance au travail. Ce qui fait dire au philosophe: «L'univers est mon repos. L'univers est ma paresse. Il n'est jamais ma pensée » (BACHELARD, 1972, p. 108).

Il faut toutefois aller plus loin. Ce n'est pas seulement l'unité de l'universmonde qui est disqualifiée par Bachelard. C'est l'unité de la pensée elle-même. Tous les lecteurs de Bachelard savent bien que son œuvre est duelle et que cette dualité repose sur celle du concept et de l'image. Toutefois il s'en faut de beaucoup que tous les lecteurs de Bachelard en tirent les conséquences qui s'imposent. Une lecture attentive des textes laisse pourtant apparaître un bilan péremptoire à cet égard ${ }^{7}$. Dès la préface de La psychanalyse du feu, le lecteur est prévenu que la poésie et la science se développent d'abord sur deux axes inverses; cependant Bachelard accorde qu'on peut peut-être les unir comme deux contraires bien faits. Plus tard cette idée de complémentarité sera même carrément abandonnée. Dans de nombreux passages de son œuvre, le philosophe distingue et sépare avec vigueur le travail du concept et la dynamique de l'imagination. Cette distinction nous vaut un bilan tardif et péremptoirement exprimé: «Entre le concept et l'image, pas de synthèse. Pas non plus de filiation (...) Qui se donne de tout son esprit au concept, de toute son âme à l'image, sait bien que les concepts et les imagesse développent sur deux lignes divergentes de la vie spirituelle» (BACHELARD, 1960, p. 46). Il est d'ailleurs assez troublant que ce texte si clair n'ait pas définitivement clôt le débat sur la soi-disant unité du bachelardisme. Car bien connue est l'affirmation qui précède, mais celle qui suit l'est beaucoup moins.

\footnotetext{
${ }^{7}$ Je crois pouvoir m'autoriser à dire que j'en ai proposé un bilan le plus exhaustif possible dans une communication faite à Cerisy-la-Salle: «Pour une éthique de la séparation: critique des herméneutiques confusionnistes", publiée dans les actes du colloque Gaston Bachelard: science et poétique, une nouvelle éthique?, sous la direction de Jean-Jacques Wunenburger (LIBIS, 2013).
} 


\section{DU MARTIN-PECHEUR A LA MANTE RELIGIEUSE \\ Jean Libis}

Bachelard a confié cecià un interlocuteur: «Ma recherche strictement scientifique n'a aucune relation avec le problème de l'imagination $»^{8}$. Et l'on le commentaire avisé de Jean Lacroix: «La conscience rationnelle et la conscience imageante appartiennent à deux mondes hétérogènes. Confondre raison et imagination, expliquer l'image à coup d'idées et l'idée à grands renforts d'images, c'est une sorte de malhonnêteté intellectuelle, destructrice de la poésie autant que de la science »(LACROIX, 1962).

Il est troublant que tout un courant exégétique ait persisté à traquer dans le bachelardisme une unité cachée. Comme Bachelard l'a abondamment développé: la séduction par l'idée d'unité est certes difficilement répressible. De ce point de vue, Roger Caillois, force est de le reconnaître, demeure volontiers dans l'entre-deux et cherche une harmonie globale qu'il ne trouve pas. Il ne s'agit pas de lui reprocher. Car d'une part il n'est jamais tout à fait dupe de lui-même. D'autre part il s'autorise à des rapprochements parfois étonnamment féconds. La mante religieuse est un de ces objets naturels qui semble tisser un pont entre la sphère des hommes et celle des insectes. À cet égard elle représente une hésitation de la raison, dont la vertu suggestive n'en est pas moins sidérante.

\section{UN PESSIMISME ONTOLOGIQUE ET UNE PHILOSOPHIE MELANCOLIQUE}

Bachelard et Caillois peuvent encore être comparés, sans qu'il y ait toutefois de confusion possible, sur le terrain d'une problématique philosophique capitale: celle du rapport au monde dans son amplitude qualitative. Plus simplement parlant: celle du rapport à la question fondamentale du pessimisme.

Pierre de Boisdeffre déclare sans ambages: «Le pessimisme de Caillois était évident. C'était même un pessimisme radical » (BOISDEFFRE, 1981, p. 53). Caillois, nous l'avons vu, a parcouru un monde profus et foisonnant dont il renonce pour finir à saisir l'unité problématique et constamment remise en question. Si les pierres ne mentent pas, l'écriture des pierres reste pourtant sans syntaxe assignable. Mais si le spectacle indéfiniment réitéré du monde naturel produit tout à la fois des moments de

\footnotetext{
${ }^{8}$ Cf. Christofides (1962).
} 


\section{DU MARTIN-PECHEUR A LA MANTE RELIGIEUSE \\ Jean Libis}

stupeur et d'enchantement, il en va différemment du monde de l'histoire dans lequel le genre humain est engagé à part entière. Or ce monde ne requiert nullement l'adhésion de l'auteur. Dans Le rocher de Sisyphe, Caillois montre sans complaisance comment une civilisation, celle d'Athènes, engendre sa propre disparition devant les roueries et les violences alternées des «barbares» macédoniens. On peut supposer que l'auteur pense aussi à l'attitude générale des démocraties occidentales face à la stratégie cynique de l'Allemagne hitlérienne. Mais il n'a pas davantage de sympathie pour le marxisme politique, dans lequel il diagnostique ce que Raymond Aron appellera l'opium des intellectuels. Il pressent que l'optimisme marxiste est partie prenante d'une idéologie dans laquelle l'Histoire moderne va se fourvoyer jusqu'au désastre.

Avec la parution tardive de son chef d'œuvre, Le fleuve Alphée, Caillois semble avouer les implications résolument non-humanistes de sa pensée tardive. Ce livre puissamment original s'apparente à un règlement de compte ourdi contre toutes les lourdeurs de la culture et la surabondance des livres médiocres qui asphyxient la vie. On retiendra quelques affirmations décapantes: «Aujourd'hui je ressens une indifférence croissante qui tantôt m'effraie et tantôt me soulage à l'égard de l'univers du savoir et de la réflexion »(CAILLOIS, 1978, p. 178). Et il ajoute presque aussitôt: «Parmi mes propres livres, il en est que littéralement je n'ose plus ouvrir ». Enfin les philosophes sont égratignés crûment, d'une manière que Bachelard n'aurait pas désavouée, dans une phrase terrible: «Comme les idées n'ont pas de volume et n'occupent aucun espace, on imagine mal que leur fourmillement tire à conséquence »(CAILLOIS, 1978, p. 181). Sont ainsi fustigés les abscons, les abstraits, les bavards. Ainsi la culture ne nous sauve pas de notre condition, et déjà dans la passé, Caillois a décoché ses flèches contre les poètes $^{9}$ (c'est un point sur lequel Bachelard adopte une attitude totalement différente, tant il est résolument accueillant aux publications parfois les plus modestes).

Même les voyages ne le passionnent plus. Ils n'ont jamais passionné Gaston Bachelard qui est l'homme du gîte, du refuge, de la maison, de l'intimité, de ce qu'il nommera tardivement «la table d'existence». Bachelard le rêveur, Bachelard le philosophe, éprouve le besoin de se protéger du monde: c'est un thème récurrent dans la partie imaginaire de son œuvre. Pessimiste, Bachelard? Certainement pas à la façon de

\footnotetext{
${ }^{9}$ Sur ce point il est plaisant de lire «L'effet Caillois » (OSTER, 1981).
} 


\section{DU MARTIN-PECHEUR A LA MANTE RELIGIEUSE \\ Jean Libis}

Roger Caillois, mais, subtilement, d'une autre manière. Si le versant épistémologique de Bachelard reste résolument pénétré d'une sorte d'enthousiasme positiviste, l'exploration de l'imaginaire va développer de tout autres tonalités. L'ouvrage sur l'eau déploie des pages troublantes sur l'affinité de l'eau, de la mélancolie et de la mort. Du reste, le surgissement d'une conscience mélancolique est récurrent dans l'œuvre ultérieure du philosophe. Elle se complique d'un rapport au vertige, particulièrement présent dans l'épisode de la cathédrale de Strasbourg ${ }^{10}$, et corroboré par cette étrange affirmation: «Toutes les idées ont leur abîme ». Il faut lire aussi de très près la méditation, explicitement placée sous le signe de Schopenhauer, éditée à la fin du volume intitulé Le droit de rêver (BACHELARD, 1970, p. 233sq.). La place nous fait ici défaut pour commenter ces textes magnifiques.

Là où Caillois explore une sorte de désenchantement fondamental tributaire d'une perte du sens, Bachelard tisse entre ses pages une mélancolie sourde et souvent pudique. Faut-il relier cette tendance à sa propre biographie, marquée précocement par la mort de sa jeune femme? Ou faut-il élargir le débat dans un sens proprement métaphysique? Comme Caillois, il ne se réfugie pas dans un sens de l'Histoire, et le politique ne sollicite pas sa réflexion philosophique ${ }^{11}$. Il est d'ailleurs symptomatique que le thème de la solitudeprenne dans son œuvre une importance croissante, y compris dans le dernier ouvrage où elle se teinte d'une lueur quasi mystique. On peut certes objecter que les dernières poétiques sont une invitation à habiter le monde. Et cela est vrai quoique le monde dont il parle soit celui d'une nature rurale recomposée par la poussée de l'imagination, par ce qu'il nomme la rêverie. Le cosmos de Bachelard est un cosmos résolument tissé par l'imagination rêveuse qui toutefois prend constamment appui sur le monde des choses mêmes: le sentier, le buisson, le ciel bleu, l'alouette. Ce monde n'est pas celui du fantastique: c'est celui de l'enfance, désancré de la question philosophique du mal et de la tragédie de l'Histoire. Ce qui ne veut certainement pas dire que son auteur en soit complètement dupe. Pas plus que Roger Caillois, il n'écrit une Théodicée.

\footnotetext{
${ }^{10}$ Il faut absolument lire cet épisode dans le chapitre XII de La terre et les rêveries de la volonté. La méditation qui suit le récit est non moins essentielle (BACHELARD, 1947).

${ }^{11}$ Cela ne signifie pas que Bachelard se désintéresse de la politique ni de son actualité, comme l'indique à plusieurs reprises la correspondance avec Marius Filloux. Mais elles demeurent essentiellement extérieures à son œuvre.
} 
Jean Libis

\section{CONCLUSION}

La confrontation entre les auvres de Gaston Bachelard et celles de Roger Caillois n'a pas la prétention de dégager une conclusion déterminante. Ces deux penseurs si complètement originaux sont sévères avec les philosophes qui s'abandonnent à l'abstraction et au prestige des idées pures. Extraordinaires explorateurs des choses mêmes qui nous entourent, ils le font avec des moyens différents. Caillois va au fin fond de la Patagonie pour découvrir une minede quartz rose et Bachelard lit surtout les poètes, attablés à sa table d'existence. L'un et l'autre sont hantés par le règne des images mais la première partie de l'œuvre de Bachelard est d'abord une apologie appuyée de la science contemporaine. L'un et l'autre ne sont pas enclins à se laisser bercer par des idéologies consolatrices ni par un humanisme facile. Il serait peut-être possible de dire que Caillois va plus loin que Bachelard dans la réduction du sens et la reconnaissance de processus régressifs. Dans Le mythe et l'homme, il affirme que le monde tend vers l'uniformité et vers la régression. C'est aussi ce qu'il décrypte dans le spectacle hallucinant d'un rivage battu par les vents au sud de la Patagonie. Bachelard au contraire n'a jamais cessé d'opposer à un destin mélancolique la nécessité d'une éthique du redressement. Il n'empêche que la lutte contre ce destin de mélancolie ne prend jamais fin. Dans un texte tardif, Bachelard évoque le souvenir d'un trait bleu entre les deux rives de la rivière de sa rivière: un martin-pêcheur qu'il n'a jamais revu, et dont l'image le hante. Au terme de cette évocation, il nous livre cette étonnante sentence métaphysique: «Le martin-pêcheur est un oiseau sans fonction temporelle, sans fonction cosmique et le voici dans deux jets d'images devenu un être d'exception temporelle, un être d'exception cosmique » (BACHELARD, 1988, p. 70). De la mante religieuse au martin-pêcheur, nous avons affaire, avec des significations différentes, à la protestation de deux penseurs profondément originaux contre la pédanterie des ratiocinateurs. 


\section{DU MARTIN-PECHEUR A LA MANTE RELIGIEUSE \\ Jean Libis}

\section{RÉFÉRENCES}

BACHELARD, Gaston. La formation de l'esprit scientifique: contribution à une psychanalyse de la connaissance objective. Paris: J. Vrin, 1938.

. Lautréamont. Paris: José Corti, 1939.

La philosophie du non. Paris: PUF, 1940.

L'eau et les rêves: essai sur l'imagination de la matière. Paris: José Corti, 1942.

La psychologie de la pesanteur. In: La terre et les rêveries de la volonté. Paris: José Corti, 1947. ch. XII, p. 341 et suivantes.

.La poétique de la rêverie. Paris: PUF, 1960.

. Fragment d'un journal de l'homme. In: . Le droit de rêver. Paris: PUF, 1970. p. 233 et suivantes.

Univers et réalité. In: L'engagement rationaliste. Paris: PUF, 1972. p. 103 et suivantes.

Fragments d'une poétique du feu. Paris: PUF, 1988.

BOISDEFFRE, Pierre de. Roger Caillois et le langage des pierres. In: GASCAR, Pierre et al. Roger Caillois ou la traversée des savoirs. 11e année. Paris: SUD, 1981. (Hors série).

BRIDET, Guillaume. Littérature mondiale et littératures nationales entre cases noires et blanches (ou case grise). Littératures, Toulouse, n. 68, p. 79-94, 2013.

CAILLOIS, Roger. Cases d'un échiquier. Paris: Gallimard NRF, 1970. La condition végétale. In: Le fleuve Alphée.Paris: Gallimard, 1978. chap. 6.

Le fleuve Alphée.Paris: Gallimard, 1978. 


\section{DU MARTIN-PECHEUR A LA MANTE RELIGIEUSE \\ Jean Libis}

CHRISTOFIDES, C.G. Bachelard's aesthetics. The journal of aesthetic and art criticism, Denver, v. 20, n. 3, 1962, p. 263 et suivantes.

LACROIX, Jean. Gaston Bachelard est mort. Le Monde, Paris, 18 oct. 1962.

LIBIS, Jean. Pour une éthique de la séparation: critique des herméneutiques confusionnistes. In: GASTON BACHELARD: SCIENCE ET POETIQUE, UNE NOUVELLE ETHIQUE?, 2012, Cerisy-la-Salle. Actes... Paris: Hermann, 2013. p. 543 et suivantes.

MORELLO, André-Alain. Une des récompenses de la rêverie. Littératures, Toulouse, n. 68 , p. 9-14, 2013.

OSTER, Daniel. L'effet Caillois. In: GASCAR, Pierre et al. Roger Caillois ou la traversée des savoirs. 11e année. Paris: SUD, 1981. (Hors série). 